\title{
SOBRE EL STATUS GRAMATICAL DEL ASPECTO Y SUS RELACIONES CON LAS PROPIEDADES TEMPORALES Y ACCIONALES
}

\begin{abstract}
Nowikow Wiaczesław, Sobre el status gramatical del aspecto y sus relaciones con las propiedades temporales y accionales [About grammatical status of the aspect and its relations to temporal and actional properties]. Studia Romanica Posnaniensia, Adam Mickiewicz, University Press, Poznań, vol. XXXl: 2004, pp. 407-415. ISBN 83-232-1353-4, ISSN 0137-2475.

The aim of this paper is to present the following three questions: a) the grammatical status of the Aspect; b) the relations of this category to temporal properties (Tense); c) the relations of the Aspect to actional properties (Aktionsart). The author makes a typological-comparative analysis of the abovementioned categories and relations in the modern Spanish and Polish and cxplains the differences and coincidences between these languages in the matter of the temporality and aspectuality.
\end{abstract}

\section{INTRODUCCIÓN A LA PROBLEMÁTICA}

En la lingüística española actual hay dos posturas con respecto a la posición que ocupan las categorías de tiempo y aspecto en la descripción del sistema verbal del castellano moderno. De acuerdo con la primera, cuyos representantes son, entre otros, Rojo y Veiga (1999; también Rojo, 1990 y Veiga, 1992), el sistema del verbo español se configura sobre las características temporales de índole deíctica. Según la segunda, apoyada, fundamentalmente, por García Fernández (1998, 1999, 2000), en el análisis del sistema verbal deberían ser introducidos parámetros aspectuales, puesto que ciertas oposiciones están basadas en los contenidos propios de la categoría de aspecto. Así, este último autor postula, p. ej., que la oposición entre el pretérito (canté) y el copretérito (cantaba) es de índole aspectual, y no temporal. Cabe subrayar que, en opinión de García Fernández (ibídem), no se trata de las características propias de la llamada Aktionsart (modo de acción, modo accional, propiedades accionales, etc.), siendo el pretérito y el copretérito exponentes de dos tipos del aspecto gramatical. Esto significa que, en principio, la primera forma 
debería funcionar como marca del contenido de 'perfectividad', mientras que la segunda como unidad portadora de 'imperfectividad'.

Como es sabido, la introducción de la categoría de aspecto en el análisis de] verbo se debe a Agrell $(1908,1918)$ quien a principios del siglo pasado publicó dos monografías sobre la prefijación verbal en polaco. De modo que el aspecto tiene una larga historia en la linguística eslava, lo que no debe extrañar al poseer la mayoría de las lenguas eslavas la expresión morfológica del aspecto que suele abarcar prácticamente todos los verbos. En lenguas tales como el ruso o el polaco que disponen sólo de tres tiempos gramaticales el aspecto es la categoría clave en la organización del sistema verbal.

La introducción del aspecto en la descripción del sistema verbal del español moderno, así como en el análisis de otras lenguas románicas (véase, v. gr., Hlibowicka-Węglarz, 1998; Guzmán Tirado, Herrador del Pino, 2000), nos hace plantear toda una serie de problemas de índole teórica, sobre todo al comparar diferentes maneras de aplicación de las categorías de tiempo y de aspecto a la hora de examinar el funcionamiento de las estructuras verbales eslavas y románicas. La solución de dichos problemas depende de cómo se resuelvan tres cuestiones claves:

- la definición del status gramatical del aspecto;

- la determinación del alcance y de la naturaleza de las relaciones que mantiene éste con las categorías de tiempo, modo y Aktionsart;

- la especificación de las coincidencias y de las diferencias con respecto al funcionamiento de las categorías en cuestión en las lenguas eslavas y románicas, y en este caso concreto en polaco y en español.

Somos conscientes de que cada uno de estos problemas merecía al menos un detallado artículo de varias páginas. De modo que vamos a centrar nuestra atención en tres cuestiones:

- el status gramatical del aspecto;

- las relaciones de éste con las propiedades temporales y

- las relaciones de aspecto con las propiedades accionales.

Vamos a hacerlo desde un planteamiento tipológico-comparado a partir del análisis de las categorías en cuestión en los idiomas polaco y español.

Cabe señalar que consideramos conveniente presentar nuestras consideraciones al respecto, puesto que, en nuestra opinión, en numerosas ocasiones al emplear los mismos términos se suele hacer referencia a conceptos distintos, lo que atañe, en primer lugar, a la noción de aspecto.

\section{EL STATUS GRAMATICAL DEL ASPECTO}

Llama la atención el hecho de que varios autores al determinar el status gramatical del aspecto, recurren a criterios y conceptos de índole fundamentalmente temporal. Se define el aspecto como un tiempo interno o como una relación no 
deíctica entre dos intervalos de tiempo. Ésta es, p. ej., la postura que, siguiendo a Comrie y Klein, adopta García Fernández (1999: 170; 2000: 45). En otras palabras, se reconoce la autonomía del aspecto en tanto que una categoría gramatical, interpretándolo simultáneamente, en términos temporales. De esta manera el aspecto aparece como una subclase de la categoría de tiempo. Por otro lado, al mismo tiempo, se define el aspecto mediante nociones tales como 'habitualidad', característica propia de la Aktionsart. Lógicamente, dichos planteamientos podrían hacernos formular la siguiente pregunta: $¿$ es necesario introducir el aspecto en el análisis lingüístico como una clase gramatical autónoma?

Por supuesto, no tenemos la menor intención de negar la relación entre tiempo y aspecto. Es obvio que diversas formas de realización del proceso o acción verbal se dan en el tiempo (sobre las relaciones entre las dos categorías véase infra). Sin embargo, no es lo mismo reconocer la existencia de una coincidencia ontológiconocional que definir una categoría lingüística en términos de otra. $\mathrm{La}$ visión „temporalizada" del aspecto se encuentra también en los trabajos de los lingüistas procedentes de los países eslavos o de los especialistas de otros países que se dedican a la investigación de los sistemas gramaticales de idiomas tales como, $p$. ej., el polaco o el ruso (véase, v. gr., Desclés y Guentchéva, 1996 o varios trabajos de Karolak sobre la problemática relacionada con el aspecto recopilados en el tomo publicado en 2001). De modo que, en principio, los enfoques no dependen del lugar de procedencia o del objeto de investigación.

No obstante, en la lingüística eslava el status del aspecto a menudo se define en términos de índole no necesariamente temporal. Esto no debe extrañar, si se toma en consideración que en polaco y en ruso el aspecto se manifiesta morfológicamente de una manera generalizada ya a nivel de infinitivo. Así, en polaco jeść pomidory significa comer tomates, mientras que zjeść pomidory (fijémonos en el prefijo z-) quiere decir comer todos los tomates hasta el último. Por supuesto, por tratarse de una distinción que se actualiza mediante dos formas de infinitivo no es necesario ni conveniente recurrir al concepto de tiempo para explicar en qué consiste la diferencia de significado. En efecto: ¿cuál es la diferencia temporal entre jeść pomidory / zjeść pomidory o comer tomates / comer todos los tomates? A lo mejor hay una, es decir en el caso de zjeść y de todos los hay un límite temporal. Sin embargo, éste tiene un carácter secundario, puesto que toda realización completa del acto (¡comer todos los tomates hasta el último!) supone un límite temporal. De hecho, al escoger entre jeść / zjeść y comer tomates / comer todos los tomates hasta el último no pensamos en distinciones temporales ni nos referimos a ellas, sino que lo que nos interesa es presentar las acciones como llevadas o no hacia su término real que, como acabamos de señalar, de forma natural tiene al mismo tiempo su límite temporal. De ahí que en la lingüística eslava, para explicar las diferencias de contenido entre los aspectos perfectivo e imperfectivo, se utilicen conceptos de 'conclusión' y 'no-conclusión'. 
No obstante, hay que reconocer que estos denominadores no siempre resultan eficaces, siendo su valor explicativo muy limitado en el caso de predicados tales como ver a alguien o alegrarse alguien. En efecto, ¿en qué consiste la conclusión del acto (proceso, acción) en pol. zobaczyt go / esp. lo vio o en pol. ucieszyl się I esp. se alegró? Las formas pronominales en posición de argumento go / lo, się / se no admiten interpretaciones 'conclusivas' válidas para los sintagmas comentados supra ni actualizaciones mediante cuantificadores todos los, hasta el último, etc. De modo que en semejantes casos el contenido aspectual transmitido se explica a partir de formulaciones tales como 'lograr / conseguir determinado estado / resultado' o 'realizar un cambio a consecuencia del cual se consigue un nuevo estado o resultado'. En nuestra opinión, el aspecto es una categoría morfológica cuyo contenido se ve determinado por la expresión de la 'conclusión' / 'no-conclusión' real de proceso verbal o por la realización completa / no-completa del acto. 'Real' significa en este caso que la [ \pm conclusión] o la realización [ \pm completa] no son exclusivamente temporales, sino que se refieren en primer lugar al término efectivo del proceso como tal, p. ej., zjeść pomidory (= esp. comer todos los tomates hasta el último). Cuando la lectura 'conclusiva' resulta imposible a causa de las restricciones sintáctico-semánticas impuestas por el verbo y por el elemento en posición de argumento, el aspecto no deja de desempeñar su función: el proceso sigue siendo enfocado como completo o concluso aunque no lo sea en términos ontológicos. No obstante, las categorías lingüísticas no coinciden más que en parte con los contenidos formulados en términos ontológicos.

En cambio, las unidades de una lengua, para que puedan ser reconocidas como tales, han de tener una manifestación propiamente lingüística. El aspecto eslavo cumple este requisito al poseer un enorme inventario de morfemas cuyo grado de productividad es, además, muy alto. Sin embargo, hay una particularidad importante (Grzegorczykowa, 1997: 25-26). Por un lado, el aspecto es una categoría gramatical cuyo objetivo es el de clasificar, es decir, todo verbo se caracteriza por el aspecto, igual que el sustantivo por el género. No obstante, la conjugación aspectual no existe. Esto ocurre, porque, por otro lado, los valores aspectuales de 'imperfectividad' y de 'perfectividad' se manifiestan mediante recursos no gramaticales sino léxicos. Nos referimos a la derivación mediante prefijos(pisać / napisać, esp. Escribir) y sufijos (dać / dawać, esp. dar), así como a las formaciones lexemáticas supletivas (brać / wziąć, esp. tomar, coger). El recurso más frecuente es la derivación que, como apunta Bogacki (2002: 7), según el diccionario electrónico Systran, alcanza el $90 \%$ del total de 6000 verbos polacos.

Cabe hacer también una observación de índole tipológica. Al tomar en consideración categorías gramaticales tales como aspecto, tiempo, caso y artículo, resulta que hay, grosso modo, dos tipos de lenguas: unas, con predominio del aspecto, y otras, con predominio del tiempo (véase, a este respecto, Pátrovics, 2000). Las primeras, a las que pertenecen, p. ej., el gótico, el llamado „Althochdeutsch", el ruso y el polaco, se caracterizan por la presencia de un sistema casual 
desarrollado y por la ausencia del artículo. Las segundas, cuyos representantes son, p. ej., el „Mittelhochdeutsch”, el búlgaro y el macedonio, tienen en su sistema gramatical el artículo, pero no poseen declinación. Éste es también el caso del castellano, lengua con un claro predominio de la categoría de tiempo.

En resumen, a nuestro juicio, las bases del sistema verbal español son claramente temporales, entre otros, por falta del aspecto morfológico. En cambio, la introducción del concepto de aspecto en la descripcion del verbo castellano se ve relacionada, en nuestra opinión, con la existencia de zonas limítrofes, coincidencias nocionales y contenidos contiguos entre aspecto, por un lado, y tiempo y Aktionsart, por otro. De modo que vamos a ver en qué consisten las relaciones del aspecto con las propiedades temporales y accionales.

\section{LAS RELACIONES DEL ASPECTO CON LAS PROPIEDADES TEMPORALES}

En primer lugar, se puede observar que en las lenguas eslavas el Aspecto Imperfectivo se da en el Presente: pol. piszę (también tiene formas de pasado y de futuro: pisatem y będę pisat, respectivamente). En cambio, el Aspecto Perfectivo no tiene formas de Presente, sino sólo de Pasado (napisatem) y de Futuro (napiszę). Esto quiere decir que exclusivamente el rasgo de [+imperfectividad] coincide con lo que Rojo y Veiga (1999) llaman 'simultaneidad al origen'. La característica de [+perfectividad] no lo hace, es decir, una acción perfectiva no puede ser simultánea al origen. De modo que desde una perspectiva tipológica, esto, hasta cierto punto, podría explicar la posibilidad de las matizaciones 'imperfectiva' y 'perfectiva' de los copretérito y pretérito castellanos: pues, el primero, sistemáticamente, es simultáneo (aunque, eso sí, no al origen, sino a un punto anterior a éste), mientras que el segundo nunca expresa simultaneidad con respecto al origen.

El hecho que acabamos de comentar podría sugerir un paralelismo funcional entre los aspectos en polaco y los tiempos en español. Sin embargo, éste no es más que parcial. Veamos un ejemplo. A menudo, para demostrar el carácter aspectual de la oposición copreterito $\neq$ pretérito se comparan ejemplos del tipo

(1) a. Ayer Isabel estaba en Sevilla.

b. Ayer Isabel estuvo en Sevilla.

Por ejemplo, García Fernández (1999: 170-171) considera la diferencia entre estos enunciados como aspectual, pero al mismo tiempo la explica en términos temporales (algunos deícticos y otros no) al utilizar conceptos tales como 'momento de la enunciacion', 'prolongación', etc. Creemos que este último hecho se ve relacionado con la característica semántica de estatividad del verbo estar: cualquier límite temporal significa en este caso la realización completa del acto. Cabe señalar 
que en polaco el verbo być (esp. estar o ser) al poseer sólo la forma imperfectiva, representa una excepción.

No obstante, el límite que establece la forma del pretérito no tiene que coincidir necesariamente con la conclusión real del proceso. Subrayemos que la coincidencia de estas propiedades en el ejemplo (1b) se explica por el carácter estativo del verbo en cuestión. De hecho, la diferencia más importante entre el pretérito y el copretérito formulada en términos no deícticos se refiere al establecimiento del límite temporal o a la ausencia de éste, cfr., p. ej.:

(2) Ayer llovió / llovía.

(3) Vivió / vivía en Sevilla.

pero

(4) Ayer llovió / *llovía todo el día.

(5) Vivió / *vivía 10 años en Sevilla.

En otras palabras, podríamos decir que si una acción aspectualmente perfectiva (pol. zjadlem pomidory $=$ esp. comi todos los tomates hasta el último) tiene inevitablemente un límite temporal, no ocurre lo mismo al revés. Es decir, una acción delimitada temporalmente no tiene que significar obligatoriamente la realización completa del acto. Por ejemplo, en castellano el empleo del pretérito en el enunciado

(6) Lavé tenedores, cuchillos, vasos, platos.

no implica necesariamente que se haya lavado todos los tenedores, cuchillos, etc. En cambio, la utilización del aspecto perfectivo en polaco lo hace de una manera inequívoca:

(7) Umylem widelce, noże, szklanki, talerze.

Si no fuese así, habría que añadir:

(8) Umytem..., ale nie wszystkie (esp. pero no todos).

En resumen, las lenguas en cuestión operan desde dos bases semánticogramaticales distintas:

- español: [ \pm delimitación temporal]

- polaco: [tconclusión procesal]

Entre éstas se establecen las siguientes relaciones:

conclusión procesal $\Rightarrow$ delimitación temporal

delimitación temporal $\neq$ conclusión procesal 
Cabe señalar que es verdad que a las formas aspectualmente perfectivas en polaco les corresponden habitualmente las formas del pretérito (o del antepresente) en español. Esto ocurre porque, como hemos apuntado supra, las acciones perfectivas tienen de una manera obligada límites temporales.

\section{LAS RELACIONES DEL ASPECTO CON LAS PROPIEDADES ACCIONALES}

Con respecto a las relaciones entre el Aspecto y la Aktionsart, creemos que se debería tomar en consideracion que mientras que el primero es una categoría gramatical con expresión morfológica propia exclusivamente del polaco, la segunda es una categoría de índole predominantemente semántica que caracteriza las dos lenguas en cuestión. Esta asimetría crea ciertos obstáculos para que los análisis semánticos de los verbos polacos y españoles se realicen a partir de las mismas bases formales y conceptuales. Por ejemplo, el verbo castellano entrar al suponer el límite y la finalidad de la acción denotada, desde el punto de vista de la Aktionsart, puede ser considerado como desinente o télico. En cambio, al lexema verbal español en polaco le corresponden dos formas aspectuales:

- la de aspecto imperfectivo wchodzić y

- la de aspecto perfectivo wejsć

De acuerdo con el valor desinente del verbo entrar, la acción, para que se realice, tiene que ser llevada a cabo hasta su término real, es decir físico.

En cambio, como hemos observado supra, el valor de perfectividad (pol. wejść) se actualiza a través de la realización completa del acto.

De modo que tenemos que ver con una coincidencia nocional de valores que, sin embargo, demuestra una diferencia considerable con respecto a los recursos lingüísticos utilizados para la categorización de dichos contenidos. Si el sistema dispone de determinados medios, una acción semánticamente desinente (télica, limitada) puede ser presentada como aspectualmente imperfectiva, es decir inconclusa en el proceso o sin realización completa del acto. Éste es el caso del polaco que al lado del perfectivo wejść posee el imperfectivo wchodzić (,,realizar la acción de entrar sin finalizarla"). Es evidente que el caso del español no es el mismo.

Por supuesto, a causa de la coincidencia nocional señalada (mutatis mutandis, algo semejante ocurre con los verbos permanentes o no-télicos y las formas aspectualmente imperfectivas), el aspecto y la Aktionsart demuestran afinidades con respecto a los contenidos transmitidos a través de la constitución del sintagma verbal. Esto se explica también por la concomitancia entre las propiedades aspectuales y accionales. El aspecto imperfectivo es más próximo a propiedades accionales tales como 'duratividad', 'iteratividad', 'habitualidad', etc., mientras que el contenido aspectual de perfectividad puede ser asociado a las características accionales de 'resultatividad', 'puntualidad', 'semelfactividad' (una prueba de 
establecer las clases prototípicamente aspectuales a partir de su relación con los rasgos de Aktionsart se encuentra en Grzegorczykowa, 1997). De una manera indirecta lo confirma la observación hecha por Bogacki (2002: 9) quien señala que en algunos diccionarios (sobre todo más pequeños) se observa la tendencia de dar equivalentes polacos a las formas correspondientes en francés o inglés no a partir de los criterios aspectuales (,comment le feraient-ils?") sino accionales. Así, chercher se traduce como szukać y no poszukać, el verbo se trouver, como znajdować się y no znaleźć się. Además, creemos que también es significativo que no haya unanimidad al establecer y al delimitar las clases de Aktionsart ni con respecto a la repartición de verbos entre estas últimas, ni en relación con los denominadores utilizados para llamarlas. Por ejemplo, a menudo se vacila entre 'inminencial' e 'ingresivo', 'habitual' y 'frecuentativo', etc. (véase, a este respecto, p. ej., Sedano, 2000: 258, 263).

Para concluir, citemos un ejemplo que demuestra que el copretérito castellano a menudo es incapaz de transmitir el contenido propio del aspecto imperfectivo polaco (subrayemos: ¡del aspecto y no de la Aktionsart!). Así, el enunciado

(9) Już trzy razy kradli ten samochód, aż go wreszcie ukradli (ejemplo de Bogacki, 2002).

con la forma de pasado imperfectivo kradli (pol. kraść, esp. robar) no puede ser traducida al español como

(10) Ya tres veces "robaban este coche hasta que por fin lo robaron.

sino como

(11) Tres veces intentaron robar este coche hasta que por fin lo robaron (de verdad).

De modo que hay casos cuando al pretérito y al copreterito castellanos les corresponden en polaco, de manera obligatoria, los aspectos imperfectivo y perfectivo, respectivamente (véase también Nowikow, 2002 y 2003).

\section{REFERENCIAS BIBLIOGRÁFICAS}

Agrell, S. (1908), Aspektänderung und Aktionsartbildung beim polnischen Zeitworte: ein Beitrag zum Studium der indogerm. Präverbia und ihrer Bedeutungsfunktionen. Lund.

Agrell, S. (1918), Przedrostki postaciowe czasowników polskich. Materialy i Prace Komisji Językowej AU, t. 8. Krak6w.

Bogacki, K. (2002), Le traitement de l'aspect dans les traducteurs automatiques de Systran, cn A. Kacprzak (ed.), Points communs: linguistique, traductologie, glottodidactique. Lódź: Wydawnictwo Biblioteka, 7-15.

Desclés, J.-P., Guentchêva, Z. (1996), Convergences et divergences dans quelques modeles du temps et de l'aspect, en V. Koseska-Toszewa, D. Rytel-Kuc (cds), Semantyka a konfrontacja jezykowa. Warszawa: Slawistyczny Ośrodek Wydawniczy, 23-42.

García Fernández, L. (1998), El aspecto gramatical en la conjugación. Madrid: Arco/Libros, S.L. 
García Fernández, L. (1999), Sobre la naturaleza de la oposición entre pretérito imperfecto y pretérito perfecto simple, Linguística Española Actual, XXI/2, 169-188.

García Fernández, L. (2000), La gramática de los complementos temporales. Madrid: Visor Libros.

Grzegorczykowa, R. (1997), Nowe spojrzenie na kalegorie aspektu w perspekswwie semantyki kognitywnej, en R. Grzegorczykowa, Z. Zaron (eds), Semantyczna struktura slownictwa $i$ wypowiedzi. Warszawa: Wydawnictwo Uniwersytetu Warszawskiego, 25-38.

Guzmán Tirado, R., Herrador del Pino, M. (2000), Investigaciones de gramática functional: la aspectualidad en ruso y español. Granada: Universidad de Granada.

Hlibowicka-Węglarz, B. (1998), Processos de expressăo do aspecto na lingua portuguesa (algumas observações de carácter contrastivo: polaco-português). Lublin: Wydawnictwo Uniwersytetu Marii Curie Skłodowskiej.

Karolak, S. (2001), Od semantyki do gramatyki. Warszawa: Slawistyczny Ośrodek Wydawniczy, 459-652.

Nowikow, W. (2002), Acerca de la distinción entre los conceptos de (tlimitación temporal) y [\#conclusión procesal], Romanica Cracoviensia, 2, 177-186.

Nowikow, W. (2003), Sobre las propiedades temporales y aspectuales. El pretérito en la construcción „estar“ + gerundio, Studia Romanica Posnaniensia, XXIX, 197-204.

Pátrovics, P. (2000), Aspektualität - Kasus - Referentialität - Temporalität. Ihre Relationen im Deutschen und in den slawischen Sprachen, en A. Kątny (ed.), Aspektualität in germanischen und slawischen Sprachen. Poznań: Wydawnictwo Naukowe Uniwersytetu im. Adama Mickiewicza, 69-86.

Rojo, G. (1990), Relaciones entre temporalidad y aspecto en el verbo español, en I. Bosque (ed.), Tiempo y aspecto en español. Madrid: Ediciones Cátedra, S.A., 17-43.

Rojo, G., Veiga, A. (1999), El tiempo verbal. Los tiempos simples, en l. Bosque, V. Demonte (eds), Gramática descriptiva de la lengua española. Madrid: RAE, Espasa Calpe, v. 2, 2867-2934.

Sedano, M. (2000), La perifrasis de gerundio en Caracas y otras ciudades hispanohablantes, NRFH, t. XLVIII, núm. 2, 253-274.

Veiga, A. (1992), La no independencia funcional del aspecto en el sistema verbal español, Español Actual, 57, 65-80. 